\title{
A role for whey acidic protein four-disulfide-core 12 (WFDC12) in the regulation of the inflammatory response in the lung
}

\author{
Arlene M A Glasgow, ${ }^{1}$ Donna M Small, ${ }^{1}$ Aaron Scott, ${ }^{1}$ Denise T McLean, ${ }^{1}$ \\ Nicolas Camper, ${ }^{1}$ Umar Hamid, ${ }^{1}$ Shauna Hegarty, ${ }^{2}$ Dhruv Parekh, ${ }^{3}$ Cecilia O'Kane, ${ }^{1}$ \\ Fionnuala T Lundy, ${ }^{1}$ Paul McNally, ${ }^{4}$ J Stuart Elborn, ${ }^{1}$ Danny F McAuley, ${ }^{1}$ \\ Sinéad Weldon, ${ }^{1}$ Clifford C Taggart $^{1}$
}

\begin{abstract}
- Additional material is published online only. To view please visit the journal online (http://dx.doi.org/10.1136/ thoraxjn-2014-206488)

${ }^{1}$ Centre for Infection and Immunity, Queen's University Belfast, Belfast, UK

${ }^{2}$ Department of Pathology, Royal Victoria Hospital, Belfast, UK

${ }^{3}$ College of Medical and Dental Sciences, University of Birmingham, Birmingham, UK ${ }^{4}$ Our Lady's Children's Hospital Crumlin, Dublin, Ireland
\end{abstract}

\section{Correspondence to} Dr Sinéad Weldon, Centre for Infection and Immunity, Health Sciences Building, Queen's University Belfast, 97 Lisburn Road, Belfast BT9 7AE, UK; s.weldon@qub.ac.uk

SW and CCT are joint senior authors.

Received 24 October 2014 Revised 9 January 2015 Accepted 10 February 2015 Published Online First 13 March 2015

\section{ABSTRACT}

Introduction Secretory leucocyte protease inhibitor and elafin are members of the whey acidic protein (WAP), or WAP four disulfide-core (WFDC), family of proteins and have multiple contributions to innate defence including inhibition of neutrophil serine proteases and inhibition of the inflammatory response to lipopolysaccharide (LPS). This study aimed to explore potential activities of WFDC12, a previously uncharacterised WFDC protein expressed in the lung. Methods Recombinant expression and purification of WFDC12 were optimised in Escherichia coli. Antiprotease, antibacterial and immunomodulatory activities of recombinant WFDC12 were evaluated and levels of endogenous WFDC12 protein were characterised by immunostaining and ELISA. Results Recombinant WFDC12 inhibited cathepsin G, but not elastase or proteinase-3 activity. Monocytic cells pretreated with recombinant WFDC12 before LPS stimulation produced significantly lower levels of the pro-inflammatory cytokines interleukin-8 and monocyte chemotactic protein-1 compared with cells stimulated with LPS alone. Recombinant WFDC12 became conjugated to fibronectin in a transglutaminase-mediated reaction and retained antiprotease activity. In vivo WFDC12 expression was confirmed by immunostaining of human lung tissue sections. WFDC12 levels in human bronchoalveolar lavage fluid from healthy and lunginjured patients were quantitatively compared, showing WFDC12 to be elevated in both patients with acute respiratory distress syndrome and healthy subjects treated with LPS, relative to healthy controls.

Conclusions Together, these results suggest a role for this lesser known WFDC protein in the regulation of lung inflammation.

\section{INTRODUCTION}

Members of the whey acidic protein (WAP), or WAP four-disulfide-core (WFDC), family of human proteins are defined by the presence of one or multiple WAP domains-a 40-50 amino acid sequence containing eight conserved cysteine residues that fold to form four disulfide bonds. ${ }^{1}$ The most studied members of this group of proteins are secretory leucocyte protease inhibitor (SLPI) and elafin, both of which contribute significantly to the

\section{Key messages}

What is the key question?

- Does whey acidic protein four-disulfide-core 12 (WFDC12) possess innate defence properties similar to other WFDC proteins in the lung such as secretory leucocyte protease inhibitor and elafin?

\section{What is the bottom line?}

- We have functionally characterised a member of the WFDC family of proteins, WFDC12, and have shown it to possess antiprotease and anti-inflammatory activities and to be present at elevated levels in the respiratory tract of patients with acute respiratory distress syndrome.

\section{Why read on?}

- Given its properties we speculate that WFDC12 may play a role in protecting lung tissue during inflammation and infection.

innate immune response via their antiprotease, antimicrobial and anti-inflammatory activities. As a result, they have each been involved in clinical trials as potential therapeutic agents to aid the control of inflammation. ${ }^{2} 3$

The small molecular weight proteins SLPI and elafin are found constitutively expressed in lung epithelia and other mucosal surfaces, demonstrating upregulation in response to a range of proinflammatory stimuli such as bacterial lipopolysaccharide (LPS), neutrophil elastase, interleukin (IL)-1 $\beta$ and tumour necrosis factor $\alpha .^{4}$ They are also produced by inflammatory cells such as neutrophils and macrophages. ${ }^{4}$

SLPI and elafin each function as inhibitors of elastase, with elafin also inhibiting proteinase- $3 .{ }^{4}$ SLPI shows no inhibition of proteinase-3 but instead is active against cathepsin G, trypsin and chymotrypsin. ${ }^{4}$ Both proteins are bactericidal against Gram negative and Gram positive species of bacteria $^{5}{ }^{6}$ and display notable antifungal ${ }^{4}{ }^{6}$ and antiviral effects. ${ }^{7} 8$ SLPI and elafin also play 
important immunomodulatory roles through their ability to reduce the host response to LPS. This is understood to occur by both extracellular and intracellular mechanisms. ${ }^{9-12}$

The role of most other WFDC proteins remains largely unknown. Our focus here is on WFDC12 (also known as WAP2). Without the predicted signal peptide, the mature 88 amino acid protein has an estimated molecular weight of $9.7 \mathrm{kDa}$ and an isoelectric point of $5.77 .^{13}$ It has an N-terminal WAP domain and a C-terminal region showing no significant homology with any currently recognised motifs. ${ }^{13}$ The WFDC12 gene is found highly expressed in lung, skin, prostate and oesophageal tissue. ${ }^{13}$ While the murine homologue protein SWAM2 has been shown to have antibacterial properties, ${ }^{14}$ there has been no data on the function of human WFDC12 reported in the literature.

The primary aim of this research was to investigate if WFDC12 harboured host defence properties akin to SLPI and elafin. Recombinant expression and purification of WFDC12 in Escherichia coli were first optimised, followed by evaluation of the protein's antiprotease, antibacterial and immunomodulatory actions. Recombinant WFDC12 (rWFDC12) was found to inhibit activity of the serine protease cathepsin G. No antibacterial ability was observed, but rWFDC12 inhibited LPS-induced cytokine production in THP-1 monocytic cells. Similar to SLPI and elafin, rWFDC12 was found to be capable of binding the extracellular matrix protein, fibronectin, and retained its antiprotease activity. Finally, we show WFDC12 expression in human lung tissue via immunohistochemistry and quantify WFDC12 levels in supernatants from LPS-treated THP-1 cells and bronchoalveolar lavage fluid (BALF) from a range of patient cohorts.

\section{METHODS}

\section{Recombinant WFDC12 expression and purification}

The mature WFDC12 cDNA sequence was cloned by Gene Art (Life Technologies, Paisley, UK) into a pQE30 vector (Qiagen, West Sussex, UK) with a six histidine coding sequence $5^{\prime}$ to the cloning region. His-tagged rWFDC12 protein was expressed and purified as detailed in the online supplement.

\section{Protease activity assays}

Cathepsin $G$ activity was measured using the fluorogenic substrate Suc-Ala-Ala-Pro-Phe-AMC (100 $\mu \mathrm{M}$; PeptaNova, Sandhausen, Germany) as outlined in the online supplement. Neutrophil elastase and proteinase-3 activity were measured using MeOSuc-AlaAla-Pro-Val-AMC (50 $\mu$ M; Enzo Life Sciences, Exeter, UK).

\section{Radial diffusion assays}

The type strain bacteria Pseudomonas aeruginosa ATCC 27853, Staphylococcus aureus ATCC 25923 and E. coli ATCC 25922 were routinely maintained at $37^{\circ} \mathrm{C}$ on Columbia blood agar plates with 5\% sheep blood (Southern Group Laboratories, Northamptonshire, UK). Double layer radial diffusion assays were carried out as described previously. ${ }^{15}$ Bacteria were washed and resuspended in $10 \mathrm{mM}$ sodium phosphate ( $\mathrm{pH}$ 7.4) buffer before incorporation into agarose underlay gels based on the same buffer. Full details are given in the online supplement.

\section{Cell culture experiments and cytokine ELISAs}

Experiments using THP-1 cells and measurement of cytokine release were carried out as outlined in the online supplement.
Cross-linking of rWFDC12 to fibronectin and evaluation of antiprotease activity of fibronectin-bound rWFDC12

Transglutamination reactions were based on previously described methods, ${ }^{16} 17$ with minor modifications using rWFDC12 and fibronectin (see online supplement).

\section{Immunostaining of human tissue from an ex vivo lung perfusion model}

Lungs harvested from potential donors which were found to be unsuitable for transplantation were obtained from International Institute for Advancement of Medicine. Ethical approval for the use of these samples was obtained from the Queen's University Belfast School of Medicine, Dentistry and Biomedical Science Research Ethics Committee (Reference Number 12.17). The lungs were perfused ex vivo based on previously described methods. ${ }^{18}$ Briefly, the lungs were initially rewarmed over a period of $1 \mathrm{~h}$ by perfusion of the pulmonary artery using Dulbecco's modified Eagle's medium (high glucose, $4.5 \mathrm{~g} / \mathrm{L}$ ) containing 5\% albumin, which was contained in a reservoir surrounded by a heated water bath $\left(38^{\circ} \mathrm{C}\right)$. The lungs were gently inflated with CPAP $\left(10 \mathrm{cmH}_{2} \mathrm{O}, 95 \% \mathrm{O}_{2}, 5 \% \mathrm{CO}_{2}\right)$. Fresh whole human blood (anticoagulated using acid citrate dextrose) was added to the perfusate once physiological temperatures were reached, and LPS (6 mg) was then introduced intrabronchially into a preselected lobe via a PE catheter (BD, 240 tubing). Experimentation ceased $4 \mathrm{~h}$ after LPS instillation, upon which tissue samples for histology were taken from both non-LPS (control) and LPS-perfused lobes. Immunostaining methods for WFDC12 and selected cell markers (epithelial cells: pan cytokeratin, macrophages: CD68, neutrophils: neutrophil elastase) are detailed in the online supplement.

\section{Human BALF collection and measurement of WFDC12}

WFDC12 levels were evaluated in BALF samples obtained from a number of different clinical trials: Samples from patients within $48 \mathrm{~h}$ of acute respiratory distress syndrome (ARDS) onset $(n=27)$ were taken from the Hydroxymethylglutaryl-CoA reductase inhibition with simvastatin in Acute lung injury to Reduce Pulmonary dysfunction (HARP) study. ${ }^{19}$ LPS-treated healthy subjects $(50 \mu \mathrm{g}$ LPS, BALF at $6 \mathrm{~h}$ post LPS-inhalation, $\mathrm{n}=10)$ and healthy controls $(\mathrm{n}=5)$ were taken from a separate study. ${ }^{20}$ Additional healthy controls $(n=6)$ were taken from the ongoing the effect of Aspirin on REducing iNflammation in Human in Vivo Model of Acute Lung Injury (ARENA) study. Ethical approval for the use of samples from the HARP (ISRCTN70127774), LPS (ISRCTN21056528) and ARENA (NCT01659307) studies was granted by the local institution and the local research ethics committee (REC numbers 06/NIR02/77, 06/NIR02/91 and 12/NI/0082 respectively). A description of the bronchoalveolar lavage procedure, sample processing and quantification of WFDC12 levels by ELISA is outlined in the online supplement.

\section{Statistical analysis}

All data were analysed using GraphPad Prism V.5.0 (GraphPad Software, San Diego, California, USA) and are reported as mean \pm SEM. Results are representative of at least $n=3$ unless otherwise indicated. Means were compared by unpaired t test or one-way analysis of variance as appropriate with Tukey's post-hoc test. Statistical significance is indicated as: ${ }^{*} p<0.05$, $* * \mathrm{p}<0.01, * * \mathrm{p}<0.001$. 
A

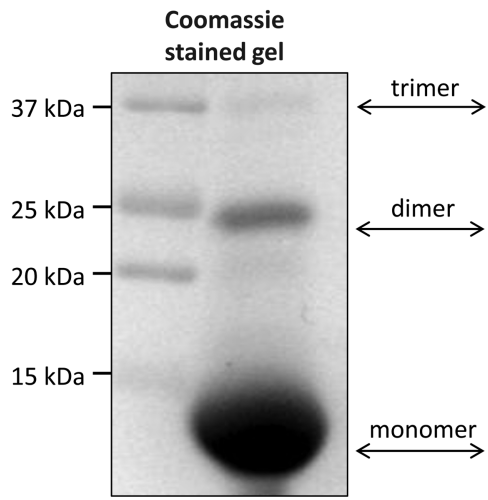

B

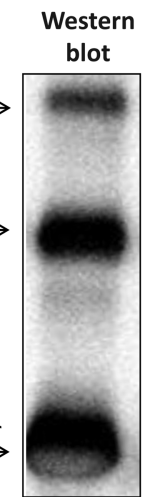

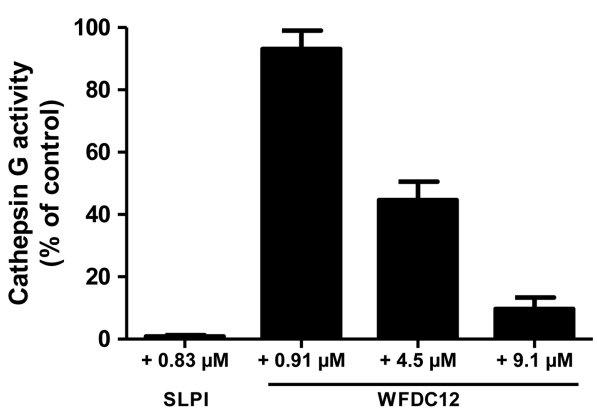

Figure 1 (A) A typical recombinant whey acidic protein four-disulfide-core 12 (rWFDC12) preparation. Induced Escherichia coli M15 were harvested and lysed in a denaturing buffer. Cleared bacterial lysate was allowed to bind Ni-NTA agarose at $4^{\circ} \mathrm{C}$ for $1-2 \mathrm{~h}$, then poured into a column and washed extensively. The eluted rWFDC12 was refolded via a step-wise dialysis protocol before analysis by Coomassie stained gel and western blot (polyclonal rabbit anti-WFDC12 antibody). (B) WFDC12 inhibits the activity of the serine protease cathepsin G. Cathepsin G (213 nM) was incubated alone or with rWFDC12 for 15 min before addition of a fluorogenic substrate (Suc-Ala-Ala-Pro-Phe-AMC). Secretory leucocyte protease inhibitor (SLPI) was used as a positive control. Results are expressed as percentage of control (untreated cathepsin G).

\section{RESULTS}

\section{Purification of rWFDC12}

The rWFDC12 used for the experiments in this study was purified under denaturing conditions as outlined in the Methods section with a typical yield of approximately $8 \mathrm{mg} / \mathrm{L}$ of culture. SDS-PAGE assessment showed the final product contained some multimers but was mostly rWFDC12 in monomeric form (figure 1A). The level of purity was reproducibly high. Identity of the purified protein was confirmed by $1 \mathrm{D}$ nLC-MS-MS (reverse phase chromatography).

\section{Antiprotease activity of rWFDC12}

Since SLPI and elafin are known to be potent inhibitors of neutrophil serine proteases in the lung, we tested rWFDC12 for similar activity. Cathepsin $G$ protease activity $(213 \mathrm{nM})$ was inhibited by rWFDC12 in a dose-dependent manner (figure 1B), with almost complete inhibition by the highest rWFDC12 amount tested $(9.1 \mu \mathrm{M})$. No significant inhibition of elastase or proteinase-3 activity by rWFDC12 was observed (data not shown).

\section{Antibacterial assays}

The WFDC proteins SLPI and elafin are reported to demonstrate notable antibacterial effects and so we investigated the ability of rWFDC12 to inhibit the growth of the type strain bacteria P. aeruginosa ATCC 27853, S. aureus ATCC 25923 and E. coli ATCC 25922. Concentrations of up to $72.7 \mu \mathrm{M}$ of protein were tested via radial diffusion assays but did not exhibit antibacterial effects (figure 2). The antimicrobial peptide cecropin A was used as a positive control.

\section{Effect of rWFDC12 on LPS challenge in monocytic cells}

The previously documented ability of SLPI and elafin to inhibit LPS-induced production of cytokines in monocytic cells led us to investigate if WFDC12 had similar properties. THP-1 monocytic cells were pretreated with rWFDC12 for $1 \mathrm{~h}$ before stimulation with LPS for $16 \mathrm{~h}$. Measurement of secreted cytokines via ELISA showed that cells pretreated with rWFDC12 had significantly lower levels of interleukin-8 (IL-8, figure 3A) and monocyte chemoattractant protein-1 (MCP-1) production (figure 3B), but not interferon $\gamma$-induced protein 10 (data not shown).

\section{Ability of rWFDC12 to bind fibronectin}

SLPI and elafin can each become cross-linked to extracellular matrix proteins, including fibronectin, in a transglutaminase catalysed reaction. To examine if WFDC12 behaved in a similar manner, rWFDC12 was incubated with fibronectin and guinea pig liver transglutaminase for $2 \mathrm{~h}$ at $37^{\circ} \mathrm{C}$. As observed by Western blot sample analysis, high molecular weight complexes $(>250 \mathrm{kDa})$ appeared when rWFDC12 and fibronectin were

Figure 2 Radial diffusion assay testing potential antimicrobial activity of whey acidic protein four-disulfidecore 12 (WFDC12). Bacteria were incubated with a range of rWFDC12 concentrations. Cecropin A and water were used as positive and negative controls respectively. $P$. aeruginosa, Pseudomonas aeruginosa; S. aureus, Staphylococcus aureus; E. coli, Escherichia coli.

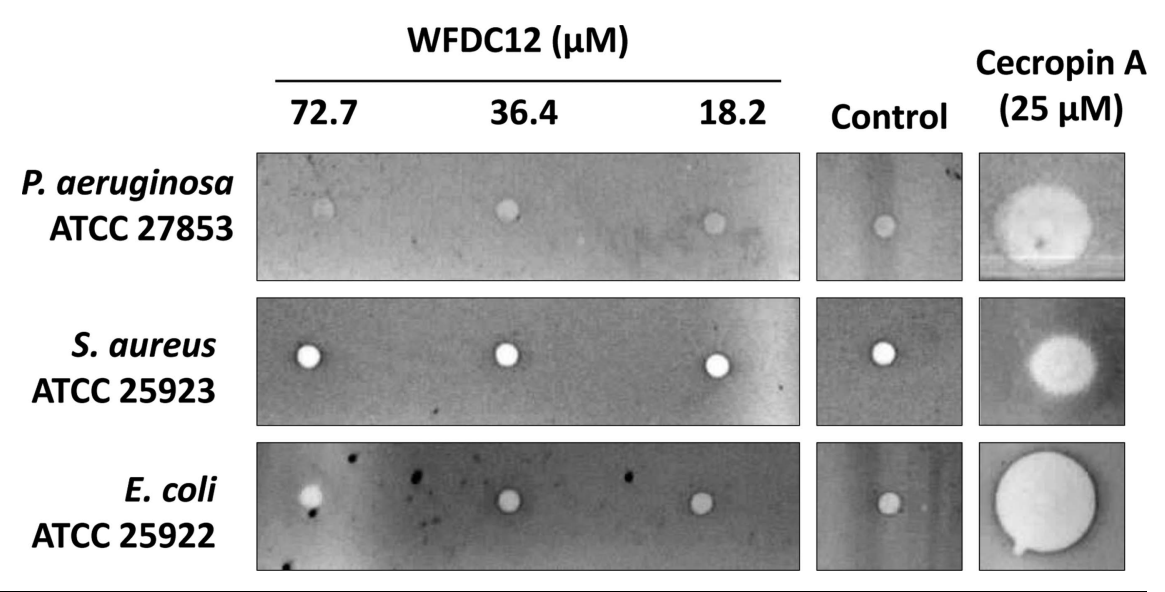

Glasgow AMA, et al. Thorax 2015;70:426-432. doi:10.1136/thoraxjnl-2014-206488 

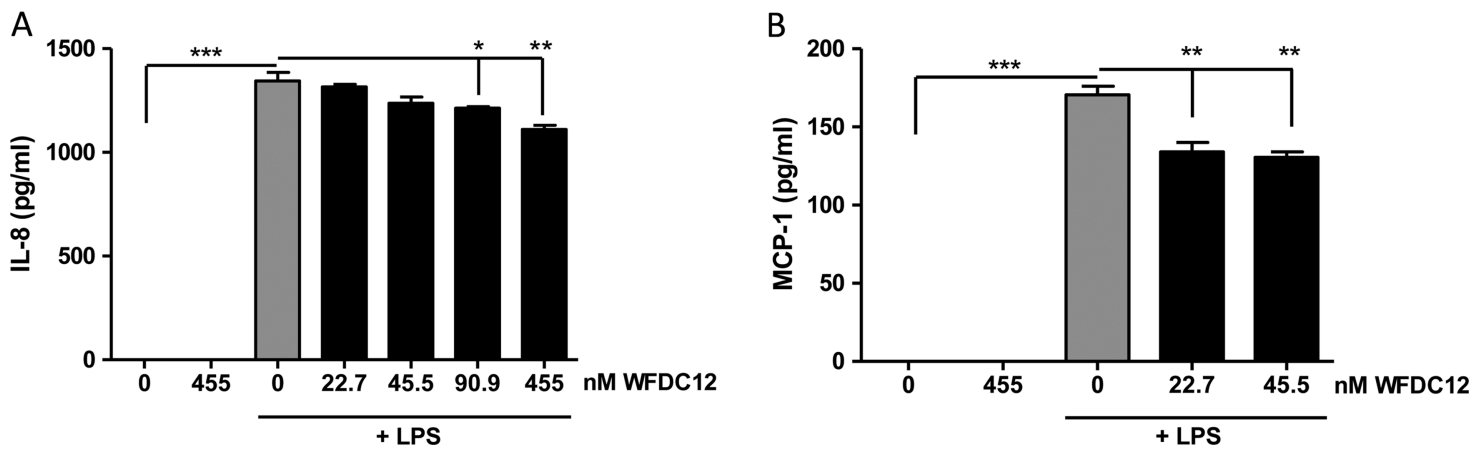

Figure 3 Investigation of potential anti-inflammatory activity of whey acidic protein four-disulfide-core 12 (WFDC12). THP-1 monocytic cells were incubated for $16 \mathrm{~h}$ with media alone (control), lipopolysaccharide (LPS) or recombinant WFDC12 (rWFDC12); or pretreated with rWFDC12 for $1 \mathrm{~h}$ before stimulation with LPS for $16 \mathrm{~h}$. (A) interleukin-8 (IL-8) and (B) monocyte chemoattractant protein-1 (MCP-1) secretion were measured by ELISA. Statistical significance is indicated as: ${ }^{*} \mathrm{p}<0.05,{ }^{* *} \mathrm{p}<0.01,{ }^{* * *} \mathrm{p}<0.001$.

incubated together in the presence of transglutaminase, but not when transglutaminase was absent (figure 4A). Accordingly, fibronectin was found depleted as visualised in a Colloidal blue stained gel (figure 4B). The heat-inactivated transglutaminase control reaction served to further certify that formation of the complexes was the result of specific enzymatic activity.

\section{Antiprotease activity of fibronectin bound rWFDC12}

In order to determine if fibronectin-conjugated rWFDC12 was still able to inhibit cathepsin G activity, rWFDC12 was added to fibronectin-coated wells of a 96-well plate, with/without transglutaminase. Unbound protein was washed away before addition of cathepsin $G$ and fluorogenic substrate and the rate of substrate hydrolysis was monitored every $60 \mathrm{~s}$ for $1 \mathrm{~h}$. Wells containing transglutaminase and rWFDC12 showed approximately $26 \%$ less cathepsin G activity compared with wells with cathepsin $\mathrm{G}$ and transglutaminase in the absence of WFDC12 (figure 4C), indicating that immobilised rWFDC12 still functioned as an inhibitor. The level of inhibition was conceivably less than that observed with $5 \mu \mathrm{g}$ of soluble rWFDC12 (figure 1B), since the cross-linking reaction is not wholly efficient. Wells containing rWFDC12 in the absence of transglutaminase showed no inhibition of cathepsin G activity (figure 4C), confirming specificity of the transglutaminase catalysed reaction.

\section{Immunostaining of WFDC12 in human tissue sections}

Human lungs rejected for transplant were perfused with LPS as outlined in the Methods section. Tissue samples from non-LPS (control) and LPS-perfused lobes were used for immunohistochemistry. Sections incubated with WFDC12 antibody showed positive staining (not detectable with isotype control) which correlated with epithelial, macrophage and neutrophil staining patterns (figure 5). WFDC12 expression was more apparent in tissue from LPS-perfused lung lobes due to increased infiltration of immune cells.

\section{Quantification of WFDC12 in THP-1 supernatants and human BALF samples}

The presence of a signal peptide suggests that WFDC12 may be secreted from cells; therefore, we used an indirect ELISA for measurement of WFDC12 in cell-free supernatants. THP-1 cells stimulated with LPS for $4 \mathrm{~h}$ showed significantly increased WFDC12 secretion (figure 6A). Furthermore, we quantified WFDC12 levels in BALF from healthy patients and patients with lung injury. BALF from patients with ARDS showed significantly increased WFDC12 relative to healthy controls (figure 6B). In addition, inhalation of LPS by healthy volunteers resulted in a significant increase in BALF WFDC12 levels compared to healthy non-LPS controls (figure 6B).

\section{DISCUSSION}

SLPI and elafin are well-characterised members of a family of proteins which each carry cysteine-rich motifs known as WFDC domains. Both SLPI and elafin have similar contributions to innate host defence via their inhibition of serine proteases, antimicrobial properties and dampening of the inflammatory response to LPS. ${ }^{21}$ In order to investigate the role of WFDC12 in similar areas of host defence, we first optimised recombinant expression and purification of the protein, then tested it for a range of functions. We found antiprotease and immunomodulatory properties, but no antibacterial activity. Furthermore, the antiprotease action of rWFDC12 was preserved when it was immobilised to fibronectin.

Although SLPI is composed of two WAP domains, its antiprotease activity has been mapped exclusively to just one of these, the C-terminal WAP domain. ${ }^{22}$ Elafin's antiprotease activity has been proven to reside in its single WAP domain. ${ }^{23}$ Recent scrutiny of WAP domains from human WAP proteins challenged the previous supposition that all of these proteins are protease inhibitors. $^{21}$ Spacing of the eight conserved cysteine residues in the domain was identified as fundamental in the determination of inhibitory potential. ${ }^{22}{ }^{23}$ The inhibitory active sites of the SLPI and elafin domains lie between cysteines 2 and 3, which are separated by eight amino acids. Among the other aligned WAP domains, this space was sometimes just three residues, leading to the proposal that, upon folding, these proteins would not contain adequate space for an inhibitory active site. ${ }^{21}$ However, the spacing within this area of WFDC12 is relatively large (seven residues), leading us to hypothesise that WFDC12 could also, like SLPI and elafin, harbour antiprotease properties. Indeed, our data confirms rWFDC12 as an inhibitor of the neutrophil serine protease cathepsin G. We speculate that the specificity of WFDC12 towards inhibition of cathepsin G, but not elastase or proteinase-3, is due to the presence of a phenylalanine residue in the putative inhibitory active site. This is a relatively large, hydrophobic amino acid which may not fit the smaller active sites of elastase or proteinase-3.

The antimicrobial activities of SLPI and elafin are associated with their cationic nature (net charges of +12 and +3 respectively) which facilitates them to disrupt the anionic bacterial cell membrane. ${ }^{6}$ However, antimicrobial peptides/proteins may also be anionic and function via alternative mechanisms. ${ }^{24}$ Of 

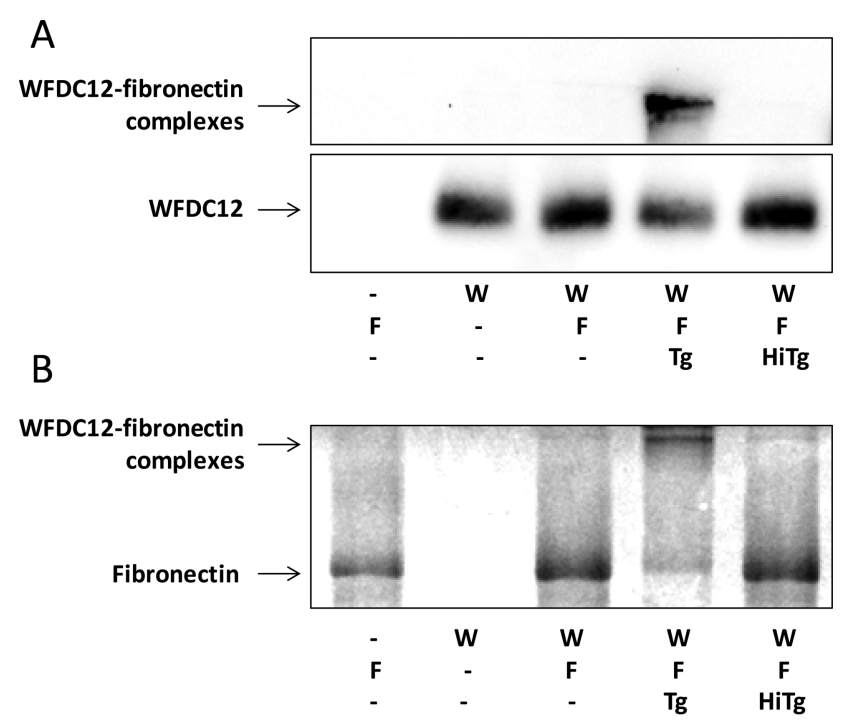

C

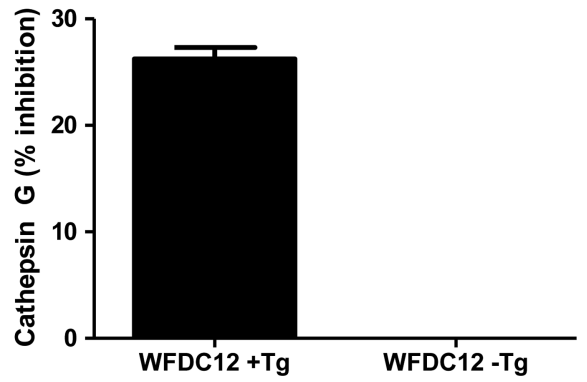

Figure 4 Whey acidic protein four-disulfide-core 12 (WFDC12) binds fibronectin via a transglutaminase-mediated reaction. Recombinant WFDC12 (rWFDC12) $(5 \mu \mathrm{g})$ was incubated with fibronectin $(20 \mu \mathrm{g})$ and guinea pig liver transglutaminase $(1 \mathrm{mU})$ in $200 \mathrm{mM}$ Tris $\mathrm{HCl} \mathrm{pH} \mathrm{7.5,}$ $20 \mathrm{mM} \mathrm{CaCl}_{2}$ and $0.1 \mathrm{mM} \mathrm{DTT}$ for $2 \mathrm{~h}$ at $37^{\circ} \mathrm{C}$. Reactions were terminated upon addition of sample treatment buffer without reducing agent, boiled and subsequently analysed by SDS-PAGE. (A) Samples were separated in both $7.5 \%$ and $15 \%$ gels, then assessed via western blot with a polyclonal rabbit anti-WFDC12 antibody. The $7.5 \%$ gel (upper panel) was used to view cross-linked protein $(>250 \mathrm{kDa}$ ) while unbound rWFDC12 was viewed on the 15\% gel (lower panel). (B) Samples were separated in a $7.5 \%$ gel and stained with Colloidal blue to allow visualisation of fibronectin and rWFDC12-fibronectin complexes. (C) WFDC12 complexed to fibronectin inhibits cathepsin G activity. Recombinant WFDC12 (4.5 $\mu \mathrm{M})$ or buffer alone was incubated with/ without guinea pig liver transglutaminase $(1 \mathrm{mU})$ in fibronectin coated wells $(20 \mu \mathrm{g} /$ well $)$ of a 96 -well plate for $2 \mathrm{~h}$ at $37^{\circ} \mathrm{C}$. Unbound rWFDC12 was washed away before addition of cathepsin $\mathrm{G}(213 \mathrm{nM})$ and subsequent incubation for $15 \mathrm{~min}$ at $37^{\circ} \mathrm{C}$. Fluorogenic substrate was then added and relative fluorescence recorded every minute for $1 \mathrm{~h}$. $\mathrm{W}=\mathrm{rWFDC12;} \mathrm{F}=$ fibronectin; $\mathrm{Tg}$ =transglutaminase; $\mathrm{Hi}=$ heat inactivated.

particular interest to us, the mouse protein SWAM2 (homologue of WFDC12), is known to be anionic and antibacterial. ${ }^{14}$ We therefore looked for similar function of WFDC12 (also anionic) against selected type strain bacteria. No apparent antibacterial effects were observed with the concentrations of rWFDC12 tested (up to $72.7 \mu \mathrm{M}$ ), however, we cannot rule out the possibility that WFDC12 may be effective against other species of bacteria.

Elafin, its precursor trappin-2, and SLPI have each been shown to become cross-linked to various extracellular matrix proteins such as fibronectin and elastin via a transglutaminasemediated reaction. ${ }^{16} 17$ They are also known to retain their antiprotease effects while immobilised, ${ }^{16}{ }^{17}$ and we have demonstrated that rWFDC12 follows suit. We hereby build upon the previously outlined theory that trapping of protease inhibitors by extracellular matrix proteins serves to protect tissue from excessive degradation by neutrophil serine proteases released during inflammation. ${ }^{16} 17$ The combination of increased exposure of extracellular matrix proteins and increased transglutaminase activity observed under inflammatory conditions is presumed to amplify conjugation of these inhibitors. ${ }^{16}$

Elafin and trappin-2 each contain one or multiple Gly-GlnAsp-Pro-Val-Lys consensus sequence motifs, a recognised substrate for the transglutaminase enzyme. ${ }^{16}$ The SLPI molecule lacks this transglutamination consensus sequence, yet also readily conjugates with fibronectin and elastin in a similar reaction. ${ }^{17}$ Mass spectrometry was used to identify the transglutaminase-reactive Gln and Lys residues within each of these three proteins. ${ }^{17}$ WFDC12 also lacks the aforementioned motif, but contains several Gln and Lys residues which could be responsible for the fibronectin-conjugation we have shown here.

We have also demonstrated the anti-inflammatory nature of rWFDC12 in its ability to inhibit LPS-induced cytokine production. Although the effect does not appear as potent as the classical examples, SLPI and elafin, we acknowledge the limitations of our recombinant protein production in E. coli. Furthermore, since LPS can activate many signalling pathways within cells, WFDC12 may target one pathway exclusively and not others, thereby limiting the degree of inhibition observed in cytokine release. With regards to the mechanism of inhibition, it is possible that WFDC12 is complexing with LPS or IL-8/MCP-1 to reduce the signal detected. However, considering that SLPI and elafin can function via an effect on intracellular signalling molecules such as $\mathrm{I} \kappa \mathrm{B} \alpha / \beta,{ }^{9}{ }^{12}$ we would anticipate that WFDC12 also has intracellular effects on LPS signalling.

We have shown in vitro that WFDC12 secretion is significantly increased in LPS-treated THP-1 cells. WFDC12 was detectable in human clinical samples by both immunohistochemistry of lung tissue sections and analysis of BALF via ELISA. The levels of WFDC12 in ARDS BALF were slightly lower than, but still comparable with, that of SLPI and elafin. WFDC12 was detected at $13.6( \pm 1.2) \mathrm{ng} / \mathrm{mL}$, equivalent to $1.24( \pm 0.11) \mathrm{nM}$; we have previously reported nanomolar levels of SLPI (approximately $8.75 \mathrm{nM}$ ) and elafin (approximately $5.11 \mathrm{nM}$ ) in BALF from patients with ARDS. ${ }^{25}$ The significantly elevated levels of WFDC12 (relative to healthy controls) found in BALF from patients with ARDS and healthy volunteers post LPS-inhalation may represent an upregulation and/or increased secretion of WFDC12 from epithelial and immune cells as part of the acute response to inflammation. Taken together, the results from this study suggest that WFDC12 may offer protective effects to the host via its antiprotease and anti-inflammatory properties.

An imbalance between endogenous proteases and their respective inhibitors is understood to be a major contributing factor to the pathology of various inflammatory lung diseases including ARDS and cystic fibrosis (CF). ${ }^{26} 27$ Antiproteases have therefore become a focal point for development of new treatments for diseases such as these, and are yielding promising results. For example, recent studies with $\alpha 1$-antitrypsin augmentation have demonstrated a reduction in protease activity, neutrophil number and pro-inflammatory cytokine levels. ${ }^{28}{ }^{29}$ SLPI is another attractive therapeutic candidate given its additional antimicrobial/antiinflammatory properties, and delivery of aerosolised SLPI showed favourable results in CF trials. ${ }^{2}$ However, deposition of the protein to poorly ventilated areas of the CF lung was later shown to be not wholly efficient. ${ }^{30}$ In addition, it is now known that both SLPI and elafin are prone to proteolytic cleavage by the excessive 


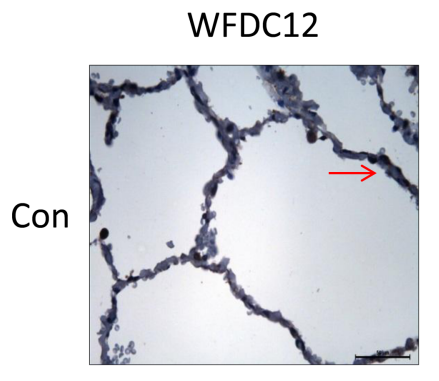

Pan Cytokeratin
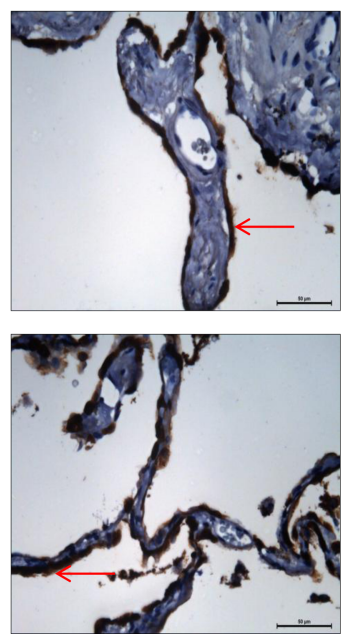

CD68
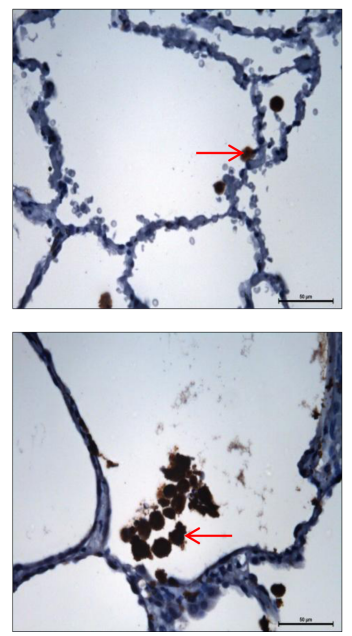

NE
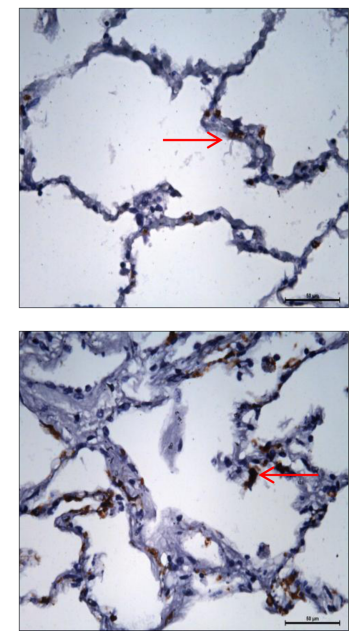

Figure 5 Expression of WFDC12 and cell specific markers in human lung tissue. Lungs deemed unsuitable for transplantation were perfused ex vivo, with lipopolysaccharide (LPS) introduced to a selected lobe for $4 \mathrm{~h}$. Tissue samples from control and LPS-perfused lobes were formalin-fixed and paraffin-embedded for immunohistochemistry. Tissue sections were stained with rabbit anti-whey acidic protein four-disulfide-core 12 (anti-WFDC12), mouse anti-pan cytokeratin (epithelial cell marker), mouse anti-CD68 (macrophage marker) and mouse anti-neutrophil elastase (neutrophil marker). Representative images $(n=2)$ were taken at $\times 40$ objective lens magnification. Brown stained cells indicate the localisation of positive staining. Scale bars shown represent $50 \mu \mathrm{m}$.

A

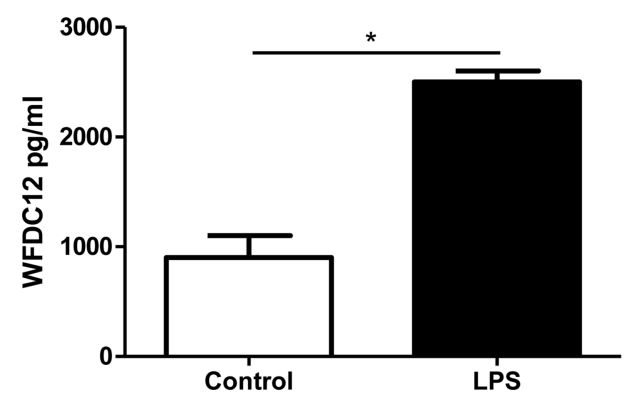

B

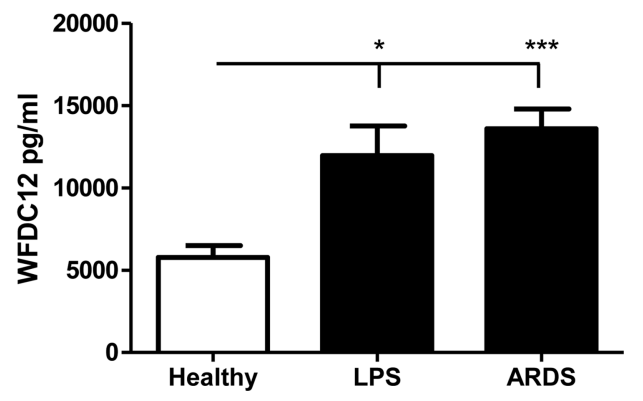

Figure 6 Quantification of whey acidic protein four-disulfide-core 12 (WFDC12) in THP-1 supernatants and human bronchoalveolar lavage fluid (BALF) samples. (A) THP-1 monocytes were stimulated with lipopolysaccharide (LPS) for $4 \mathrm{~h}$ and WFDC12 secretion measured by ELISA. (B) WFDC12 was measured by ELISA in BALF samples from the following patient groups: healthy controls $(n=11)$, healthy subjects sampled $6 \mathrm{~h}$ following LPS inhalation $(n=10)$ and patients within $48 \mathrm{~h}$ of acute respiratory distress syndrome (ARDS) onset $(n=27)$. Statistical significance is indicated as: ${ }^{*} p<0.05,{ }^{* * *} p<0.001$.

levels of proteases associated with dysregulated inflammation, ${ }^{25} 31$ perhaps limiting their full therapeutic capacity. Therefore, WFDC12 should ideally be examined for similar susceptibility before investigation as a possible therapy.

Further exploration of WFDC12 properties is needed to determine its potential as a therapeutic agent in chronic inflammatory disorders of the lung. Given that elafin inhibits elastase and proteinase-3 but not cathepsin G, combination with WFDC12 would result in the targeting of all three neutrophil serine proteases and could prove more highly effective. Furthermore, delivery of WFDC12 as a fibronectin-conjugate may offer advantages of increased bioavailability and stability in an environment laden with proteases.

Contributors AMAG, SW, AS, DMS, DTM and NC performed the experiments. UH, CK, DP, DFM, PN, JSE and FTL provided biological samples. SH carried out analysis. AMAG, SW and CCT designed experiments and drafted manuscript. All the authors reviewed the manuscript and approved its submission.

Funding This work was supported by the Department of Employment and Learning (Northern Ireland) and the Science Foundation Ireland Research Frontiers Programme (04/BR/B0640).
Competing interests None.

Patient consent Obtained.

Ethics approval ORECNI.

Provenance and peer review Not commissioned; externally peer reviewed.

\section{REFERENCES}

1 Ranganathan S, Simpson KJ, Shaw DC, et al. The whey acidic protein family: a new signature motif and three-dimensional structure by comparative modeling. J Mol Graph Model 1999;17:106-13, 134-6.

2 McElvaney NG, Nakamura H, Birrer $\mathrm{P}$, et al. Modulation of airway inflammation in cystic fibrosis. In vivo suppression of interleukin-8 levels on the respiratory epithelial surface by aerosolization of recombinant secretory leukoprotease inhibitor. J Clin Invest 1992;90:1296-301.

3 Shaw L, Wiedow O. Therapeutic potential of human elafin. Biochem Soc Trans 2011;39:1450-4.

4 Sallenave J-M. Secretory leukocyte protease inhibitor and elafin/trappin-2: versatile mucosal antimicrobials and regulators of immunity. Am J Respir Cell Mol Biol 2010;42:635-43.

5 Hiemstra PS, Maassen RJ, Stolk J, et al. Antibacterial activity of antileukoprotease. Infect Immun 1996;64:4520-4. 
6 Baranger K, Zani M-L, Chandenier J, et al. The antibacterial and antifungal properties of trappin-2 (pre-elafin) do not depend on its protease inhibitory function. FEBS J 2008;275:2008-20.

7 McNeely TB, Dealy M, Dripps DJ, et al. Secretory leukocyte protease inhibitor: a human saliva protein exhibiting anti-human immunodeficiency virus 1 activity in vitro. J Clin Invest 1995:96:456-64.

8 Ghosh M, Shen Z, Fahey JV, et al. Trappin-2/Elafin: a novel innate anti-human immunodeficiency virus-1 molecule of the human female reproductive tract. Immunology 2010;129:207-19.

9 Taggart CC, Greene CM, McElvaney NG, et al. Secretory leucoprotease inhibitor prevents lipopolysaccharide-induced IkappaBalpha degradation without affecting phosphorylation or ubiquitination. J Biol Chem 2002;277:33648-53.

10 Taggart CC, Cryan S-A, Weldon S, et al. Secretory leucoprotease inhibitor binds to NF-kappaB binding sites in monocytes and inhibits p65 binding. J Exp Med 2005;202:1659-68.

11 McMichael JW, Roghanian A, Jiang L, et al. The antimicrobial antiproteinase elafin binds to lipopolysaccharide and modulates macrophage responses. Am J Respir Cell Mol Biol 2005;32:443-52.

12 Butler MW, Robertson I, Greene CM, et al. Elafin prevents lipopolysaccharide-induced AP-1 and NF-kappaB activation via an effect on the ubiquitin-proteasome pathway. J Biol Chem 2006;281:34730-5.

13 Lundwall A, Clauss A. Identification of a novel protease inhibitor gene that is highly expressed in the prostate. Biochem Biophys Res Commun 2002;290:452-6.

14 Hagiwara K, Kikuchi T, Endo Y, et al. Mouse SWAM1 and SWAM2 are antibacterial proteins composed of a single whey acidic protein motif. J Immunol 2003;170:1973-9.

15 McLean DTF, Lundy FT, Timson DJ. IQ-motif peptides as novel anti-microbial agents. Biochimie 2013;95:875-80.

16 Guyot N, Zani M-L, Maurel M-C, et al. Elafin and its precursor trappin-2 still inhibit neutrophil serine proteinases when they are covalently bound to extracellular matrix proteins by tissue transglutaminase. Biochemistry 2005;44:15610-18.

17 Baranger K, Zani M-L, Labas V, et al. Secretory leukocyte protease inhibitor (SLPI) is, like its homologue trappin-2 (pre-elafin), a transglutaminase substrate. PLoS One 2011;6:e20976.
18 Lee JW, Fang X, Gupta N, et al. Allogeneic human mesenchymal stem cells for treatment of $E$. coli endotoxin-induced acute lung injury in the ex vivo perfused human lung. Proc Natl Acad Sci U S A 2009;106:16357-62.

19 Craig TR, Duffy MJ, Shyamsundar M, et al. A randomized clinical trial of hydroxymethylglutaryl-coenzyme a reductase inhibition for acute lung injury (The HARP Study). Am J Respir Crit Care Med 2011;183:620-6.

20 Shyamsundar M, McKeown STW, O'Kane CM, et al. Simvastatin decreases lipopolysaccharide-induced pulmonary inflammation in healthy volunteers. Am J Respir Crit Care Med 2009;179:1107-14.

21 Bingle $C D$, Vyakarnam $A$. Novel innate immune functions of the whey acidic protein family. Trends Immunol 2008;29:444-53.

22 Eisenberg SP, Hale KK, Heimdal P, et al. Location of the protease-inhibitory region of secretory leukocyte protease inhibitor. J Biol Chem 1990;265:7976-81.

23 Tsunemi M, Matsuura Y, Sakakibara S, et al. Crystal structure of an elastase-specific inhibitor elafin complexed with porcine pancreatic elastase determined at $1.9 \mathrm{~A}$ resolution. Biochemistry 1996;35:11570-6.

24 Harris F, Dennison SR, Phoenix DA. Anionic antimicrobial peptides from eukaryotic organisms. Curr Protein Pept Sci 2009;10:585-606. .

25 Kerrin A, Weldon S, Chung AH-K, et al. Proteolytic cleavage of elafin by $20 \mathrm{~S}$ proteasome may contribute to inflammation in acute lung injury. Thorax 2013;68:315-21.

26 Moraes TJ, Chow C-W, Downey GP. Proteases and lung injury. Crit Care Med 2003;31:\$189-94.

27 Cohen-Cymberknoh M, Kerem E, Ferkol T, et al. Airway inflammation in cystic fibrosis: molecular mechanisms and clinical implications. Thorax 2013;68:1157-62.

28 Griese M, Latzin P, Kappler M, et al. alpha1-Antitrypsin inhalation reduces airway inflammation in cystic fibrosis patients. Eur Respir J 2007;29:240-50.

29 Geraghty P, Rogan MP, Greene CM, et al. Alpha-1-antitrypsin aerosolised augmentation abrogates neutrophil elastase-induced expression of cathepsin $B$ and matrix metalloprotease 2 in vivo and in vitro. Thorax 2008;63:621-6.

30 Stolk J, Camps J, Feitsma HI, et al. Pulmonary deposition and disappearance of aerosolised secretory leucocyte protease inhibitor. Thorax 1995;50:645-50.

31 Weldon S, McNally P, McElvaney NG, et al. Decreased levels of secretory leucoprotease inhibitor in the Pseudomonas-infected cystic fibrosis lung are due to neutrophil elastase degradation. J Immunol 2009;183:8148-56. 